\title{
基于等离激元微纳结构的太阳能界面 光蒸汽转换
}

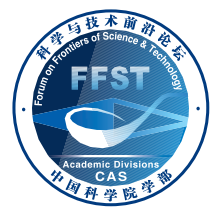

\author{
刘海舟 ${ }^{1}$, 喻小强 ${ }^{2}$, 李金䂞 ${ }^{1}$, 徐凝 ${ }^{1}$, 周林 $^{1 *}$, 朱嘉 $^{*}$ \\ 1. 南京大学现代工程与应用科学学院, 南京 210093 ; \\ 2. 东南大学物理学院, 南京 211189 \\ *联系人, 朱嘉, E-mail: jiazhu@nju.edu.cn; 周林, E-mail: linzhou@nju.edu.cn
}

收稿日期: 2019-03-06; 接受日期: 2019-04-04; 网络出版日期: 2019-07-31

国家自然科学基金(编号: 11874211)、国家重点基础研究发展计划(编号: 2015CB659300)和国家重点研发计划(编号: 2017YFA0205700)资助

\begin{abstract}
摘要 作为人工微纳结构与光热转换的融合, 太阳能光蒸汽转换成为近年来太阳能热利用领域的研究热点之一. 基于人工微纳结构的等离激元吸收体, 能够在整个太阳光谱范围内实现高效的光子捕获. 等离激元吸收体表面和 内部的光致局域加热, 促成了高效的液气相变.与碳基吸收体相比，等离激元吸收体不但可将入射太阳光局域在 亚波长尺度内, 形成致密的热点, 而且其吸收光谱可灵活调控. 围绕等离激元吸收体的光蒸汽转换体系, 还具有热 响应快、材料抗腐蚀、可回收等优势, 可用于海水淡化、污水处理、灭菌消毒等水处理领域. 本文从光吸收和热 局域两个角度阐述了等离激元光蒸汽转换系统的设计与优化, 并对其应用前景作了展望.
\end{abstract}

关键词等离激元共振, 光蒸汽转换, 微纳结构, 太阳能

PACS: $64.70 . \mathrm{Fx}, 65.80 .+\mathrm{n}, 78.67 . \mathrm{Bf}, 89.30 . \mathrm{Cc}$

太阳能转换是新能源领域的重要科学前沿. 人类 利用太阳能的形式很多, 如基于太阳能电池的光-电转 化、基于光催化过程的光-化学能转化等. 在太阳能的 诸多利用形式中, 太阳能光热转换是最古老、最直 接、最普遍的利用方式, 与人类的生活息息相关.

作为地球的终极能量来源，太阳辐射功率密度达 到 $3.828 \times 10^{26} \mathrm{~W}$. 由于受到大气层的反射、散射、吸 收等，理想情况下到达地球表面的辐射功率密度要低 很多 $\left(\sim 1000 \mathrm{~W} / \mathrm{m}^{2}\right)$. 更为重要的是, 太阳能辐射波段宽 (主要分布于280-4000 nm波段), 分布于有限波长间隔
内的太阳辐射功率密度相对较低(如在太阳辐射谱密 度最高的可见波段，500-600 nm波段的太阳辐射也只 占总太阳辐射的 $15.2 \%)$. 因此, 基于天然窄带吸收体 的太阳能光热转换的效率往往不高, 人们对太阳能的 热利用还远远不够.

太阳能光蒸汽转换, 是当今太阳能热利用的一个 新兴研究分支. 顾名思义, 太阳能光蒸汽转换是指通 过光吸收体捕获太阳能并转化为热能, 加热水体, 使 其发生液气相变转化为蒸汽. 常见的光蒸汽转换过程, 例如大气水循环中的蒸发过程，以及太阳池的光蒸馏

引用格式: 刘海舟, 喻小强, 李金砟, 等. 基于等离激元微纳结构的太阳能界面光蒸汽转换. 中国科学: 物理学 力学 天文学, 2019, 49: 124203 Liu H Z, Yu X Q, Li J L, et al. Plasmonic nanostructures for advanced interfacial solar vapor generation (in Chinese). Sci Sin-Phys Mech Astron, 2019, 49: 124203, doi: 10.1360/SSPMA-2019-0070 
过程, 都是利用太阳辐射直接进行体相加热. 近年来发 展的基于人工微纳结构的太阳能光蒸汽转换，具有以 下优势：（1）对全波段太阳光的吸收更加充分 $\left(>95 \%{ }^{[1,2]}\right)$ ；(2) 局域空间加热，提高了加热效率; (3) 基于人工微纳结构的光热调控，可以在提高光子捕获 效率的同时，有效抑制辐射、对流以及无效热传导等 热学损失. 这些优势使得吸收的太阳能绝大部分转化 为水体蒸发过程中的焓变, 能量转换效率大为提高(在 无聚焦、无绝热、1个太阳强度下达到 $80 \%$ 以上 ${ }^{[3]}$ ). 因 此, 人工微纳结构光热材料是高效太阳能光蒸汽产生 的关键. 研究表明，等离激元微纳结构具有快速热响 应和优异的空间热局域特性，是光蒸汽转换的理想材 料之一, 成为了近年来太阳能光蒸汽转换研究的热点.

20 世纪初, Wood ${ }^{[4]}$ 在研究金属光栅时发现衍射异 常. 随后，此现象被证明是金属-电介质分界面的表面 电磁模式被激发引起的，也被称为表面等离极化激元 (Surface Plasmon Polariton, SPP) ${ }^{[5]}$. 自1998年Ebbesen 等人 ${ }^{[6]}$ 报道亚波长金属孔阵结构的增强光学透射效应 以来, 等离激元微纳结构所具有的灵活光学响应, 以及 突破光学衍射极限的光传输能力，吸引了广泛的研究 兴趣. 等离激元微纳结构常被用于亚波长尺度的电磁 能量聚焦, 在微纳激光器 ${ }^{[7]}$ 、表面增强拉曼散射 ${ }^{[8]}$ 、 电磁隐身 ${ }^{[9]}$ 等领域中逐渐得到应用.

作为信息载体，等离激元光学结构往往需要克服 热损耗. 反之, 作为能量载体, 等离激元结构的热效应 却有其用武之地. 基于等离激元结构的热利用, 已开始 应用到太阳能转换、光电催化、光热治疗等领域. 等 离激元光蒸汽转换是其中一个重要的研究方向. 2013 年, 美国莱斯大学Halas研究组利用金纳米颗粒产生高 温蒸汽 ${ }^{[10]}$. 此后, 等离激元光蒸汽转换与应用引起了 诸多研究者的兴趣. 在新型太阳能利用领域，等离激 元材料成为了除碳基材料之外的一个重要研究分支. 它不仅能够实现吸收/辐射光谱的有效裁剪，而且通过 与光的相互作用, 形成亚波长尺度的局域热点. 超薄的 等离激元吸收体, 在太阳光谱的主要辐射波段, 具有近 似完美吸收的水平 ${ }^{[11]}$. 与碳基材料相比, 在可回收性、 抗腐蚀性等物化性质上, 也有一定优势 ${ }^{[12]}$.

我们首先回顾了表面等离极化激元的基本概念, 重点关注与光蒸汽转换直接相关的性质. 接着, 从光学 吸收和热局域两个角度阐述了等离激元光蒸汽转换的 设计思想. 最后, 我们将介绍等离激元光蒸汽转换的具
体应用领域, 并展望其应用前景.

\section{1 等离激元简介}

传统各向同性的体块电介质材料，其色散一般表 现为 $k=n \omega / c$, 其中 $n$ 为介质折射率, $c$ 为光速. 这将导致 态密度 $D(\omega)$ 呈现出 $\omega^{2}$ 的分布 ${ }^{[13]}$. 这种分布模式中, 太 阳波段处的光子态密度相对较低(图1(a)), 极大限制了 体块电介质的太阳光吸收. 而等离激元材料借助光学 共振，能够激发特定波长处态密度的尖峰，从而增大 太阳波段的光子吸收率.

\section{1 表面等离极化激元与局域表面等离激元}

表面等离极化激元最早发现于金属-电介质界面, 因此狭义的表面等离极化激元又称金属表面等离极化 激元. 这是由自由电子的集体振荡与电磁波之间相互 耦合形成的一种极化激元，发生在金属与电介质界面， 沿着垂直于边界的方向迅速衰减.

图1(b)展示了表面等离极化激元的色散曲线. 随 着切向波矢的增大, 光子频率逐步逼近 $\omega_{\mathrm{SPP}}=$ $\omega_{\mathrm{p}} / \sqrt{\varepsilon_{\infty}+\varepsilon_{\mathrm{m}}}$ (其中 $\omega_{\mathrm{p}}$ 为体块等离子振荡频率， $\varepsilon_{\infty}$ 为金 属的高频介电常数, $\varepsilon_{\mathrm{m}}$ 为电介质的介电常数 $)^{[14]}$, 这使 得 $\omega_{\mathrm{SPP}}$ 处的光子态密度远超其他频率. 因此, 材料表面 的自由电子振荡能够与该频率的入射光发生强烈耦 合, 实现共振吸收. 如今, 表面等离极化激元吸收体在

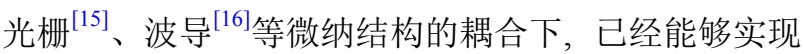
接近 $100 \%$ 的吸收峰.

与表面等离极化激元类似，局域表面等离激元 (Localized Surface Plasmon, LSP)源于光与金属颗粒 表面自由电子集体振荡的耦合. 在Mie散射理论中, 由 于边界条件的限制，本征模式由连续解转变为离散的 级数解, 表现为不同角动量量子数 $l$ 所对应的共振模式 的叠加(图1(c) $)^{[17]}$. 通常当颗粒的尺寸远小于波长时, 只有 $l=1$ 的模式较为显著, 对应 $\varepsilon=-2 \varepsilon_{\mathrm{m}}$ 处的Frohlich窄 带共振吸收 ${ }^{[18]}$. 对于非球形颗粒的局域表面等离激元, 则需通过更为普适的Gans理论 ${ }^{[19]}$ ，或有限时域差分 ${ }^{[20]}$ 等数值手段进行研究.

局域表面等离激元显著增大了材料本身的吸收截 面. 以金属银为例，单颗粒表面的光场增强可达 $10^{3}$ 倍. 随着两颗粒间距的减小，其间隙处的光场增强可高达 $10^{6}$ 倍 ${ }^{[21]}$. 共振伴随着强烈的欧姆损耗，这使得金属颗 

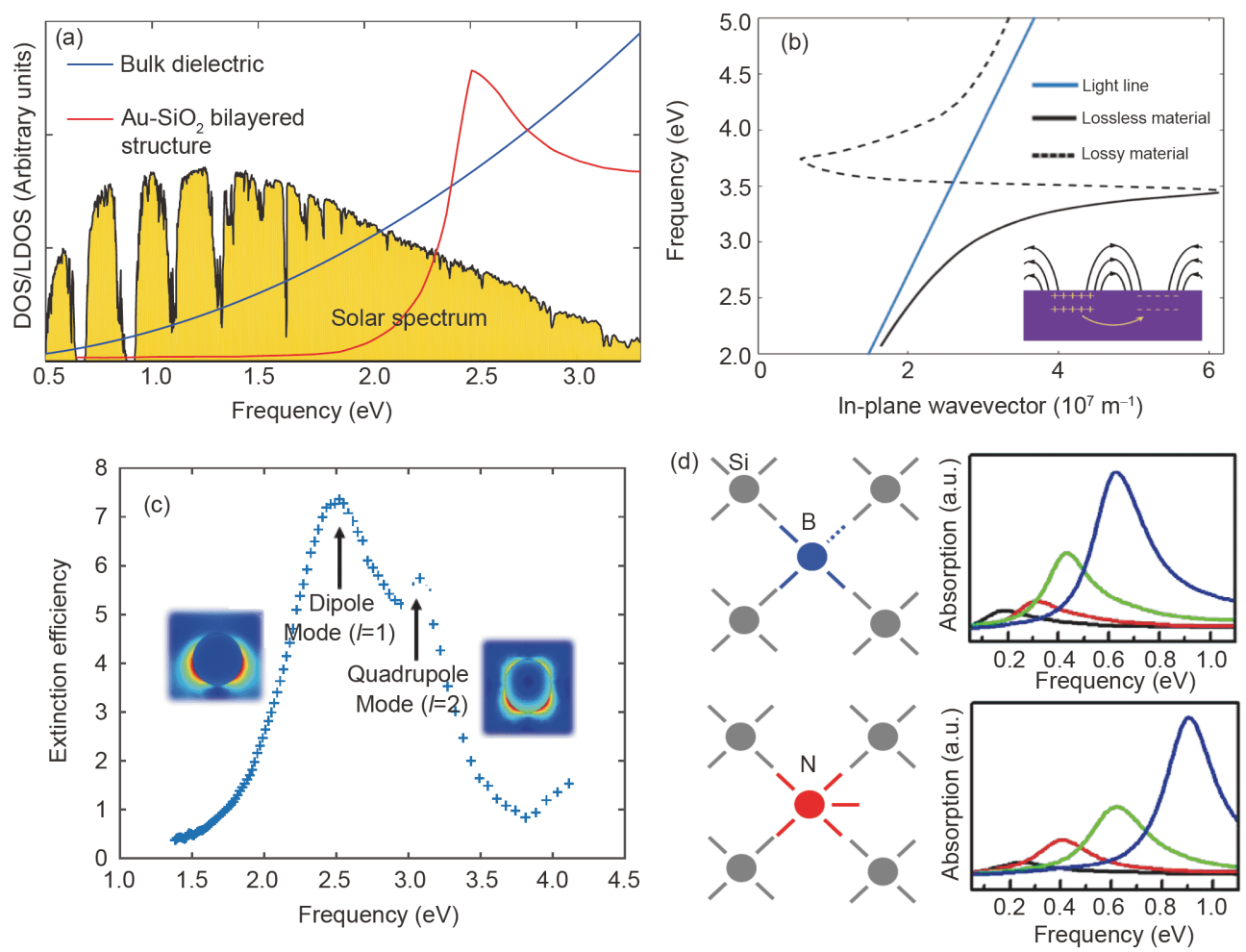

图 1 (网络版彩图)等离激元效应. (a) 体块电介质(蓝)与金-二氧化硅分界面(红)的态密度/局部态密度对比. 太阳光的频谱以 黄色填充标注出, 作为参考. (b) 银-二氧化硅分界面的色散曲线. 黑色实线、虚线分别代表理想金属、和具有欧姆损耗的金属 的色散曲线. 体块电介质中的光锥线以蓝色实线标出. 插图展示了等离激元模式. (c) 半径50 nm银颗粒的消光效率谱. 图中两 个峰分别对应 $l=1$ 的偶极子模式和 $l=2$ 的四极子模式. 数据与电场分布图像取自文献 $[17]$. (d) 高掺杂半导体中掺杂 $\mathrm{p}$ 型半导体 (硼)及 $\mathrm{n}$ 型半导体(磷)的等离激元效应 ${ }^{[22]}$. 黑、红、绿、蓝曲线分别对应 $2.5,5,10,20 \times 10^{20} \mathrm{~cm}^{-3}$ 的掺杂浓度

Figure 1 (Color online) Plasmonic resonances. (a) Comparison of dispersion relations of a bulk dielectric (blue) and an $\mathrm{Au}_{-} \mathrm{SiO}_{2}$ interface (red). Solar spectrum as a function of photon energy is shaded in yellow for reference. (b) Density of state/local density of state at the metal-dielectric interface. Solid and dashed lines in black respectively represent lossless and lossy metals. The light line in bulk dielectrics is sketched in blue. The inset shows the diagram for surface plasmons. (c) Extinction efficiency of a $50 \mathrm{~nm}$ silver nanoparticle derived for Mie's theory. Peak 1 and peak 2 respectively represent the dipole mode $(l=1)$ and quadrupole mode $(l=2)$. Data and figures for electric field distribution are extracted from Ref. [17]. (d) Plasmonics in heavily-doped semiconductors, classified into p-type (boron injected) and n-type (phosphorous injected) [22]. Black, red, green and blue curves represent doping densities of $2.5,5,10,20 \times 10^{20} \mathrm{~cm}^{-3}$, respectively.

粒在共振波段具有较强的吸收特性。与SPP主导的微 纳结构相比，由随机取向的纳米颗粒组装而成的结构， 具有天然的对入射光的偏振方向和入射角度的不敏感 特性. 另外, 纳米颗粒具有较大的表体比, 能够与流体 介质充分接触, 提高热转移本领, 在等离激元光蒸汽转 换系统中具有一定优势.

\section{2 等离激元共振的调控}

与碳基体系相比，通过等离激元模式构建的光热 体系，其吸收体厚度显著减小．碳基吸收体厚度一般 达到微米级别，而等离激元材料具有极小的光学趋肤 深度(银的趋肤深度在 $100 \mathrm{~nm}$ 以下), 这将带来材料用
量的减少和体系便携性的提升.

等离激元作为一种结构驱动的光吸收模式，与体 块材料本身的吸收性质具有较大区别. 尺寸、构型、 背景电介质的变化都会使共振峰产生定向移动，而颗 粒构型也会影响到吸收截面. 图2(a)-(d)展示了金纳米 颗粒的吸收截面随上述参数的变化规律. 多元化的结 构调控手段为精准的吸收光谱裁剪提供了有效手段. 因而我们能控制特定波段处的光吸收性能.

本征吸收材料的吸收模式受比尔-朗伯定律的约 束, 具有单一的电磁场分布模式. 而基于等离激元的光 热转换结构, 可以根据光学热点的实际布局需要, 相对 自由地控制其区域和程度. 作为例证, 图2(e)对比了体 

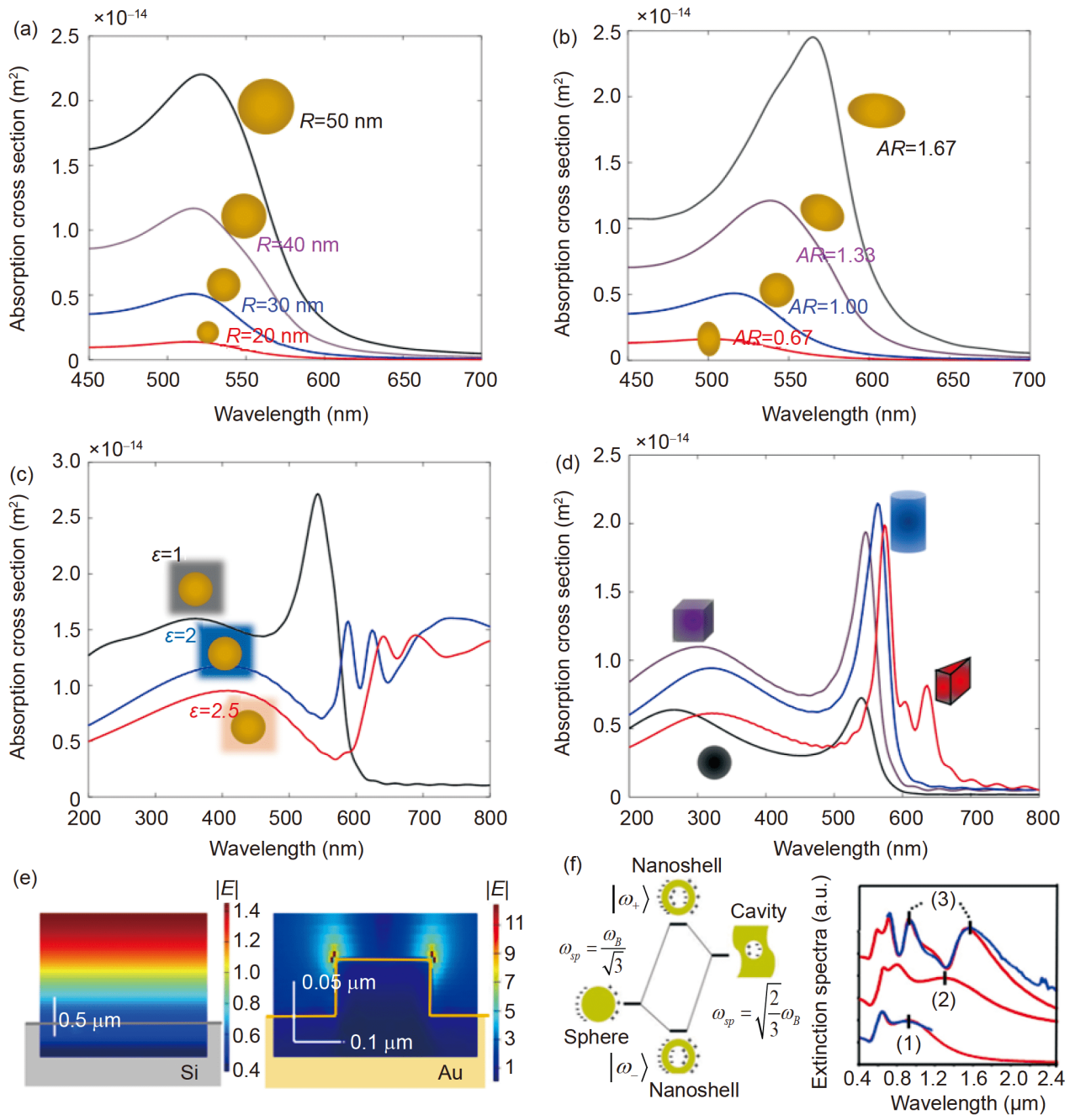

图 2 (网络版彩图)等离激元的光学调控及杂化性质. (a)-(d) 金纳米球等离激元模式的影响因素. 由尺寸(a)、长短轴比(b)、 背景介电常数(c)、几何构型(d)导致的吸收截面变化. (e) 本征半导体 $\mathrm{Si}$ (左)与光栅结构(右)在入射光照射下的电场分布曲线. (f) 纳米壳中的等离激元模式杂化 (量子数 $l=1$ 的情形 $)^{[23]}$. 位于 $\omega_{\mathrm{B}} / \sqrt{3}$ 的球模共振峰与位于 $\sqrt{2 / 3} \omega_{\mathrm{B}}$ 的腔膜进行杂化(左), 形成 对称的 $\left|\omega_{-}\right\rangle$和反对称的 $\left|\omega_{+}\right\rangle$. (1), (2), (3)分别对应内层球腔、外层球、整个纳米壳的消光谱

Figure 2 (Color online) Optical regulation and hybridization properties of plasmonics. (a)-(d) Impact factors of plasmonic properties for gold nanoparticles. Absorption cross sections of various particle sizes (a), aspect ratios (b), background indices (c), and geometries (d) are plotted. (e) Electric field distributions for intrinsically absorbing silicon (left) and a grating structure (right). (f) Plasmonic hybridization in nanoshell structures (Case where quantum number $l=1$ ) [23]. Hybridization of sphere mode $\omega_{\mathrm{B}} / \sqrt{3}$ and cavity mode $\sqrt{2 / 3} \omega_{\mathrm{B}}$ is achieved, forming a symmetric mode $\left|\omega_{-}\right\rangle$ and antisymmetric mode $\left|\omega_{+}\right\rangle$. Plots labeled (1), (2), and (3) respectively refer to extinction spectra for inner cavity, outer sphere and the whole nanoshell.

块硅和金纳米光栅的电场分布曲线. 热点区具有更强 的电场强度和更强的热转化本领. 在光蒸汽转换中, 我们通常需要将热点集中于空气与水的分界面，从而 最大程度减小向水体的热传导损失.

近来, 研究表明, 通过半导体掺杂也可以使得半导 体具有等离激元效应. 半导体颗粒的LSP及其光热效
应也引起了研究者的兴趣. 掺杂过程可引入大量的自 由载流子, 使半导体的带隙变窄而具有类金属的特性. 通过调控杂质浓度, 可灵活调节半导体中的等离激元 特性. 美国凯斯西储大学Burda与南京大学朱俊杰课题 组 ${ }^{[24]}$ 合作，报道了类 $\mathrm{p}$ 型半导体 $\mathrm{Cu}_{2-x} \mathrm{~S}$, 发现其具有类 LSP效应，并利用Mie散射公式推得化学计量数 $2-x$ 与 
消光系数的关系. 美国莱斯大学Nordlander课题组 ${ }^{[22]}$ 通过随机相位近似法，计算了硅中的 $\mathrm{p}$ 型掺杂和 $\mathrm{n}$ 型掺 杂带来的吸收截面移动(图1(d)).

\section{3 等离激元杂化与耦合}

2003年，美国莱斯大学的Prodan等人 ${ }^{[23]}$ 提出了等 离激元杂化的概念，完美解释了纳米同心球壳中的共 振模式(图2(f)). 球壳被看作是大半径的球体与小半径 的球形腔的叠加. 文章指出，对于服从Drude模型 $\varepsilon(\omega)$ $=1-\omega_{\mathrm{P}}{ }^{2} / \omega^{2}$ 的金属, 共振频率为 $\omega_{\mathrm{P}} \sqrt{(l+1) /(2 l+1)}$ 的 球模与频率为 $\omega_{\mathrm{p}} / \sqrt{2 l+1}$ 的腔模进行杂化，形成成键 态耦合模式 $\left|\omega_{-}\right\rangle$和反键态耦合模式 $\left|\omega_{+}\right\rangle$. 一系列不同半 径的同心球壳组合而成的结构，将具有更多的等离激 元杂化模式. 相关研究进一步表明，大量金属颗粒之 间的杂化，能够克服等离激元窄带吸收的固有缺陷， 具备选择性宽带吸收的特征 ${ }^{[11]}$, 从而完全有可能满足 太阳能光蒸汽转换对于吸收体的光谱要求(本文中所 指的宽带吸收, 如不另外提及, 均指太阳波段内的宽带 吸收).

\section{2 光蒸汽转换的物理机理与性能评估}

理想的太阳能光蒸汽转换要求尽可能全波段吸收 太阳光, 并充分转化为目标液体的焓变, 这其中要经历 复杂的光物理和热学过程. 接下来我们对光蒸汽转换 的微观和宏观机理进行阐释，给出影响蒸发效率的重 要因素.

\section{1 光蒸汽转换的微观和宏观机理}

等离激元微纳结构在将光能转换为热能, 实现水 的气液相变的过程中, 宏观上要经历光吸收、热弛 豫、界面传热、相变等一系列物理过程, 相应地在微 观上经历光激发热载流子产生、热载流子衰减、热耗 散 ${ }^{[25]}$ 等(图3(a)). 吸收体通过内部光子、电子、声子的 相互作用, 实现光热转化及热量传递.

（1）光激发. 以金属纳米颗粒为例，当入射光频率 与局域表面等离激元的共振频率较为接近时，等离激 元表面电子的强烈振荡使得电场发生扭曲. 坡印亭矢 量由外部指向纳米颗粒 ${ }^{[26,27]}$, 增大了颗粒的有效吸收 截面. 在 $100 \mathrm{fs}$ 的时间尺度内, 电子间相互作用, 使体 系产生新的热载流子. 在能带图中表现为电子穿越费 米面，打破稳态下的费米-狄拉克分布. 研究表明 ${ }^{[28]}$, 电子-空穴对的产率与纳米颗粒的尺寸及材料的载流 子寿命密切相关. 因此, 光激发过程能够通过对纳米颗 粒的宏观尺度调控和材料选择来实现.

（2）衰减过程. 表面辐射衰减作为吸收的逆过程, 导致了体系能效比的降低，但吸收体内部的热辐射可 导致光线在结构内部的散射. 非辐射衰减在朗道阻尼 机制的作用下，促成了绝大部分的光热转换. 在 $1 \mathrm{ps}$ 的时间内载流子完成弛豫过程, 通过电子-电子、电 子-声子散射过程，热载流子的热运动动能被重新分 配, 逐渐弛豫到晶格振动的光学声子能量, 再通过多 声子散射过程实现到低频率声学声子的弛豫. 在这个 过程中, 光能被有效转化为载流子热运动的动能并最 终转化为低频声子的振动能, 宏观上表现为吸收体内
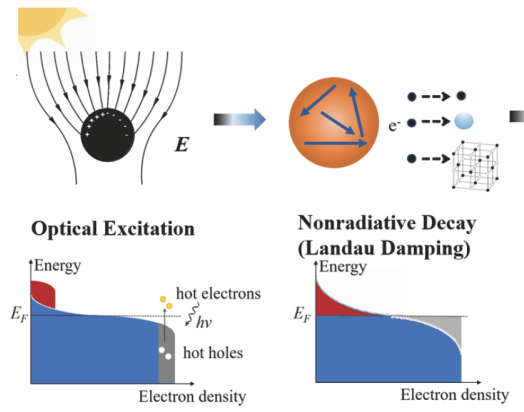

(a)

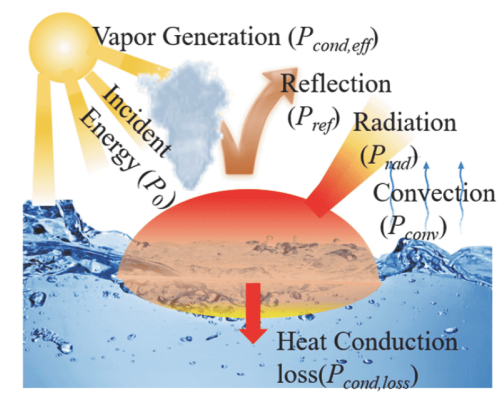

(b)

图 3 (网络版彩图)光蒸汽转换的微观、宏观过程. (a) 光蒸汽转换的三个阶段: 光激发、非辐射衰减、热耗散; (b) 光蒸汽转 换的宏观过程. 入射能量转化为反射、辐射、传导、对流等形式

Figure 3 (Color online) Process of solar vapor generation in the microscopic and macroscopic picture. (a) Three phases of solar vapor generation from the microscopic picture: optical excitation, nonradiative decay and thermal dissipation; (b) the macroscopic picture of solar vapor generation. Incident energy is converted into such forms as reflection, radiation, conversion and convection. 
能的增加.

(3) 热耗散. 低频声子振动能通过吸收体声子和水 分子振动耦合(宏观上表现为热传导、热对流等方式) 实现能量到液态水的传递. 持续的光能辐照最终实现 吸收体温度与外部环境的局域动态热平衡，特征时间 为0.1-10 ns. 液态水分子吸收热量, 克服氢键和范德 瓦尔斯作用力, 转化为气态分子并离开体相. 这一过 程建立稳定热平衡状态的时间，对体系外部环境的依 赖性很大, 往往会达到宏观时间尺度量级.

从宏观层面上看, 光蒸汽转换是一个输入与输出 功率维持动态平衡的过程, 如式(1)所示:

$P_{0}=P_{\text {ref }}+P_{\text {rad }}+P_{\text {cond }}+P_{\text {conv }}$,

其中, $P_{\mathrm{ref}}$ 为吸收体反射功率; $P_{\mathrm{rad}}$ 为吸收体再辐射功 率; $P_{\text {conv }}$ 为水体与空气的对流功率; $P_{\text {cond }}$ 为吸收体的总 热传导功率, 包括了有效热量传导 $P_{\text {cond,eff }}$ 和传导损失 $P_{\text {cond,waste }}$ 两个部分, 分别对应着吸收体提供水体焓变 的能量和加热体块水的能量. 近年来兴起的界面光蒸 汽转换(Interfacial Solar Vapor Generation, ISVG) ${ }^{[29]}$, 正是由于对体块水传热的高度抑制，而实现较高的蒸 汽产生速率.

\section{2 光蒸汽转换性能评估}

光蒸汽产生效率可通过计算蒸汽带走热量占总入 射能量的比重得到:

$\eta=\dot{m} h_{\mathrm{LG}} / P_{\text {solar }}$,

其中, $h_{\mathrm{LG}}$ 为水蒸发焓, $P_{\text {solar }}$ 为标准太阳光辐射强度. $\dot{m}$ 指水蒸发速率，严格来说是真实蒸发速率 $\dot{m}_{\text {solar }}$ 减去 暗场蒸发速率 $\dot{m}_{\mathrm{dark}}$. 在光蒸汽转换中, 传递给蒸汽的 热量是唯一有效的传导热量 $\left(P_{\text {cond,eff }}=\dot{m} h_{\mathrm{LG}}\right)$. 高效的 蒸汽产生可通过两种方式实现: 增大光吸收和增大有 效热传导在能量再分配中的比重.

增大光吸收, 要求吸收体应具备对于整个太阳光 谱区 $0.28-4 \mu \mathrm{m}$ 接近于 $100 \%$ 的吸收率. 有效吸收率可 以定义为

$\alpha_{\text {eff }}=\frac{\int_{\text {Solar spectrum }} A(\lambda) I_{\text {solar }}(\lambda) \mathrm{d} \lambda}{\int_{\text {Solar spectrum }} I_{\text {solar }}(\lambda) \mathrm{d} \lambda}$,

其中, $A(\lambda)$ 为吸收体在 $\lambda$ 波长处的吸收率, $I_{\text {solar }}(\lambda)$ 为 $\lambda$ 波 长处的太阳光强度. $\alpha_{\mathrm{eff}}$ 是衡量吸收体水平的重要依据. 经过长期探索, 在金属和碳基材料中, 已经能够普遍实
现超过 $>95 \%$ 的有效吸收率.

为了增大有效热传导在光蒸汽转化中的比重, 我 们需要着重考察吸收体的热辐射、热传导、热对流等 因素. 对热辐射的研究可通过考察红外热辐射率来 表征:

$\varepsilon_{\text {eff }}(T)=\frac{\int_{\lambda_{1}}^{\lambda_{2}} A(\lambda) I_{\text {blackbody }}(\lambda, T) \mathrm{d} \lambda}{\int_{\lambda_{1}}^{\lambda_{2}} I_{\text {blackbody }}(\lambda, T) \mathrm{d} \lambda}$.

一般地， $\varepsilon_{\text {eff }}$ 越低，表明辐射损失被抑制得越充分. 这里的 $\left[\lambda_{1}, \lambda_{2}\right]$ 为吸收体的主要辐射区间, 其具体取值 因吸收体的具体工作温度决定. 对于光蒸汽转换, 因 吸收体温度较低，其辐射波段和太阳光谱区几乎无交 叠. 因而理想吸收体的吸收带边, 应位于 $4 \mu \mathrm{m}$ 和 $\lambda_{1}$ 之间 (图4(a)). 另外, 对太阳标准光谱文件ASTM G-173-03 的简单分析可知, 近 $99 \%$ 的太阳辐射落在 400-2500 nm 波段, 所以有时也可以将 $2500 \mathrm{~nm}$ 作为吸收带边的极 限值.

式(1)中，热传导、热对流项分别由傅里叶定律、 牛顿冷却定律来决定:

$$
\begin{aligned}
& q_{\mathrm{cond}}=-\kappa_{T} \nabla T, \\
& q_{\mathrm{conv}}=h\left(T-T_{\mathrm{amb}}\right),
\end{aligned}
$$

其中, $q_{\mathrm{cond}}$ 和 $q_{\mathrm{conv}}$ 分别为热传导和热对流的热流密度, $\kappa_{T}, h$ 分别为热传导和热对流系数, $T, T_{\mathrm{amb}}$ 分别为体系 和环境温度. 降低系统与环境的温差, 能够有效减小传 导、对流和辐射能量损失, 提高光蒸汽转换效率. 另 外, 可以利用热传导方程(式(6)), 定量计算传导、对流 等热学损失.

$$
\begin{aligned}
& \rho(\mathbf{r}) c(\mathbf{r}, t) \frac{\partial T(\mathbf{r}, t)}{\partial t} \\
& \quad=\nabla \cdot[k(\mathbf{r}, t) \nabla T(\mathbf{r}, t)]+P(\mathbf{r}, t),
\end{aligned}
$$

其中, $t, \mathbf{r}$ 分别为观测点的时空坐标; $\rho, c, k, T, P$ 分别指 质量密度、热容、热导率、温度、输入功率. 通过计 算得到的温度分布图, 可以定量分析热局域的程度、 热能的传递过程和能量损失的大小, 给材料、结构的 优化带来极大便利.

\section{3 等离激元光蒸汽转换结构的微纳设计}

实现高效光蒸汽转换的关键因素有: (1) 全波段太 阳光的吸收; (2) 局域的热学调控. 这两个因素都依赖 
于等离激元结构的具体构建形式, 例如材料、构型, 以 及采用的外部辅助手段(如祄底、系统结构等).

\section{1 等离激元宽带光吸收体设计}

基于金属颗粒的光子纳米流体是最早实现等离激 元光蒸汽转换的方式之一. 美国莱斯大学的Halas课题 组 ${ }^{[10]}$ 在对等离激元光蒸汽系统的探索中, 将尺寸均一 的 $\mathrm{Au} @ \mathrm{SiO}_{2}$ 核壳颗粒散布于水中(图4(b)). 通过对颗粒 尺寸的优化, 金颗粒的吸收谱与太阳光谱尽量交叠, 整 个流体体系因而能较为充分地利用太阳光.

使用纳米流体构建吸收体，存在一些固有的缺点. 例如, 金纳米颗粒在多次蒸汽循环后容易聚集成团, 导 致等效的颗粒尺寸改变, 吸收效果也相应减弱. 上海交 通大学邓涛课题组 ${ }^{[30]}$ 从材料制备的角度进行了有效 的改进，提出在无尘纸上沉积纳米金颗粒的实验方案， 并报道了 400-800 nm波段> $80 \%$ 的光吸收效率和 4.5 倍 太阳下 $77.8 \%$ 的光热转换效率. 由于纸的表面粗糙度
高，表面反射被降低，沉积在无尘纸上的金膜(非离散 的金属颗粒)比平整的金膜或金颗粒纳米流体具有相 对高的吸收效率. 而与纳米流体相比, 能实现 $25 \%$ 的蒸 汽产率提升. 值得一提的是, 这一报道的巨大效率提升 可能不仅与光吸收有关，也更依赖于整个复合结构对 热传导损耗的抑制. 阿卜杜拉国王科技大学的Fratalocchi课题组 ${ }^{[12]}$ 从纳米颗粒结构上对吸收体进行了优 化, 将纳米球改为纳米球-纳米棒复合结构. 该类结构 能够将入射光聚焦于球与棒的接触部位，并转换为热 能, 在太阳光谱区其平均吸收率高达 $91 \%$.

与 $2 \mathrm{D}$ 组装相比, 3D组装结构不仅在体系上的多重 散射更具优势, 同时也支持更多的等离激元模式. 南京 大学朱嘉课题组 ${ }^{[11]}$ 利用多孔阳极氧化铝模板(Anodic Aluminum Oxide, AAO), 进行金纳米颗粒的三维自组 装, 制备出吸收率高达 $99 \%$ 的近完美吸收体. 通过大量 颗粒间的等离激元共振模式杂化(图4(c)), 吸收体克服 了单一金属纳米颗粒的窄带吸收特征，将吸收带扩展 (a)

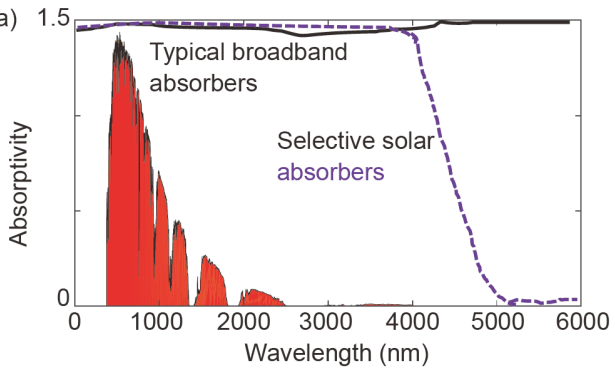

(c)

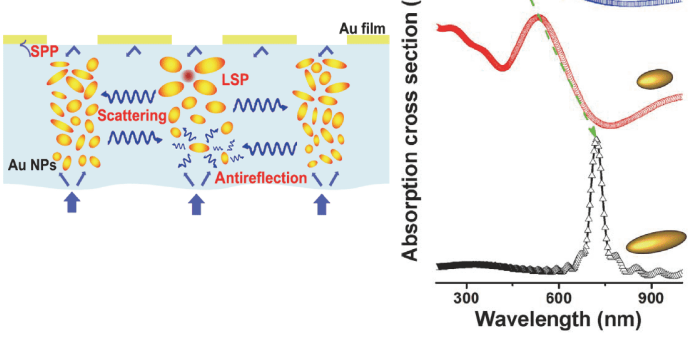

(b)
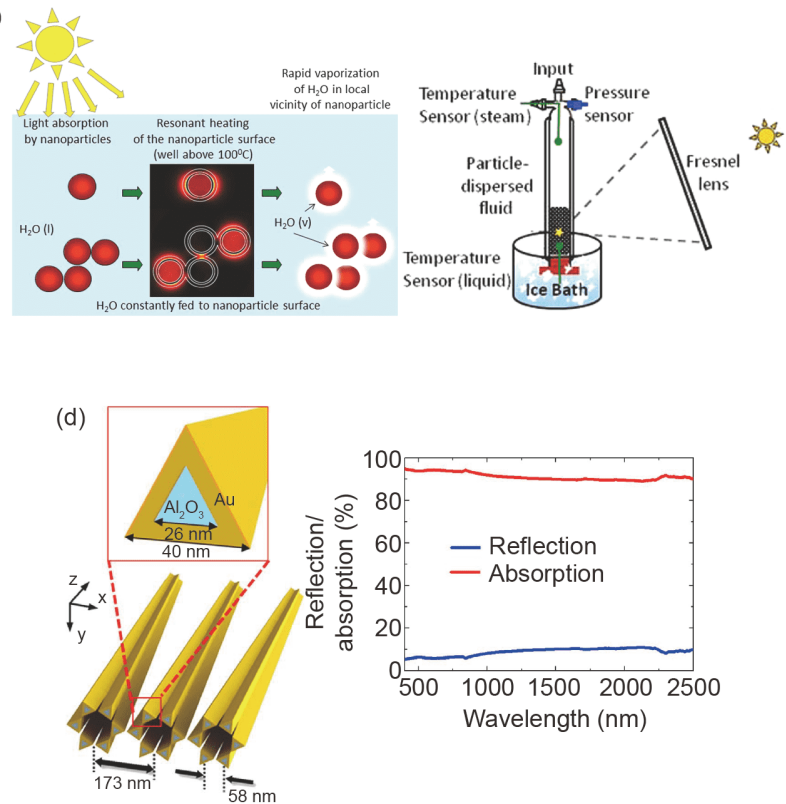

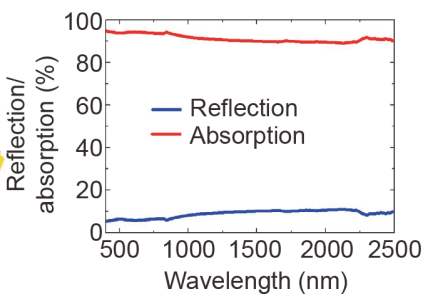

Wavelength $(\mathrm{nm})$

图 4 (网络版彩图)光蒸汽转换吸收体的理想吸收谱及具体的宽带吸收体设计. (a) 典型宽带吸收体(黑色实线)与选择性吸收 体(紫色虚线). (b) Halas课题组提出的用金纳米流体实现高效光蒸汽转换的方案 ${ }^{[10]}$. 左图: 金纳米颗粒利用太阳能示意图. 右 图: 光蒸汽转换的具体实验装置. (c) 南京大学朱嘉课题组构建的AuNP-AAO宽带吸收体 ${ }^{[11,31]}$ (左). 不同长短轴比的椭球体具有 不同的光学模式(右) ${ }^{[31]}$, 在同一体系中的混合杂化能够使吸收体具有宽带的吸收. (d) Bae 等人利用间隙等离激元模式构建的 黑金薄膜(左), 实现了高达 $91 \%$ 的宽带太阳光吸收(右) ${ }^{[2]}$

Figure 4 (Color online) Ideal absorption spectrum for solar thermal evaporators, and corresponding broadband absorption designs. (a) Typical broadband absorbers (black solid line) and selective absorbers (purple dashed line). (b) Solar vapor generation enabled by gold nanoparticles, proposed by the Halas group [10]. Left: Schematic for AuNP-triggered phase change. Right: Concrete system established for solar evaporation. (c) Absorber design combining AuNPs and AAO, proposed by the Zhu group (left) [11,31]. Ellipsoids with various aspect ratios have different optical modes (right), and thus equip the absorber with broadband absorption [31]. (d) Bae et al. [2] took advantage of gap plasmon modes to construct black gold membranes (left), realizing high solar absorption as much as $91 \%$ (right). 
至中红外波段. AAO也可辅助构建与空气匹配的折射 率, 通过减小界面阻抗，有效抑制反射. 美国马里兰大 学胡良兵课题组 ${ }^{[32]}$ 进而改为选用更为廉价的木材作 为基质材料组装金颗粒. 由于木材的孔洞具有更大的 直径 $(5-50 \mu \mathrm{m})$, 因而吸收体能通过波导模式束缚不同 波长的入射光, 实现高达 $99 \%$ 的平均吸收率, 吸收带边 也扩展至 $50 \mu \mathrm{m}$.

出于对光学性质和化学稳定性的考虑, 当今的等 离激元光蒸汽系统大多选用金纳米颗粒作为吸收体材 料. 新型的吸收体设计, 在材料、结构上进行了一些创 新. 例如, 通过结构调控, 部分课题组将铝 ${ }^{[33]}$ 、铟 ${ }^{[34]}$ 、 氮化铁 ${ }^{[35]}$ 等材料的吸收共振峰移动至太阳光波段. 值 得一提的是, 中山大学杨国伟课题组 ${ }^{[36]}$ 创新性地提出 将窄禁带半导体碲的纳米颗粒用于光蒸汽转换，利用 碲的光学二重性实现宽带高吸收. 在太阳光谱范围内, 随着波长的增加，碲纳米颗粒的介电常数由负变化到 正，实现金属属性向全介质属性的转变. 通过结合等 离激元共振和介质的Mie共振，具有宽粒径分布的碲 纳米颗粒可以覆盖整个太阳光谱，实现 $85 \%$ 的平均吸 收率. $\mathrm{Ba} \mathrm{e}^{\text {等人 }}{ }^{[2]}$ 构建了顶角低至 $1^{\circ}$ 的尖锐凹槽阵列, 利用绝热纳米聚焦的方式，成功制备出在太阳光谱区 高达 $91 \%$ 的吸收率的黑金薄膜(图4(d)). 此类雉形结构 有效抑制了光线的反射. 不同波长的入射光进入结构 后，会停留在间隙中不同深度的位置，耦合形成间隙 等离激元模式, 最终通过欧姆损耗转换为热能.

\section{2 等离激元驱动的高效热学调控}

到目前为止, 等离激元吸收体的性能, 已经非常接 近理想吸收体. 但光蒸汽转换并不仅仅依赖吸收体的 完美吸收, 这也表明高效光蒸汽产生与体系的热学调 控密切相关. 对热传导、热对流等热学损失项进行针 对性的抑制, 能够使得吸收体吸收的能量最大限度用 于水的蒸发焓变.

高浓度的金属纳米颗粒，具有天然的热局域本领， 自发抑制对整个水体的加热. 金属的高消光系数 $\kappa$ 本身 带来较小的趋肤深度，而高颗粒浓度的金属纳米颗粒 又进一步提高了热局域程度，实现更强、更聚集的热 点. 美国莱斯大学Halas课题组 ${ }^{[37]}$ 通过计算高浓度金纳 米颗粒中的场分布，发现颗粒的多重内部散射能够显 著增加光程，突破比尔-朗伯定律预测的光子吸收效 率. 局域光吸收和热传递, 造成水体顶部和底部 $25^{\circ} \mathrm{C}$
的温差，光热转换被高度局限在 $\sim 0.5 \mathrm{~mm}$ 深的水体表 面(图5(a)). 邓涛课题组 ${ }^{[38]}$ 在此研究基础上做了改进, 在水体中混入 $10 \mathrm{~nm}$ 金颗粒和 $500 \mathrm{~nm}$ 聚苯乙烯颗粒 (图5(b)). 聚苯乙烯颗粒有效抑制了金颗粒的背向散 射，使得整个散射、吸收过程被进一步局域，最高可 实现 $54.7 \%$ 的蒸发效率提升.

随着界面光蒸汽研究的不断深入，采用大孔隙率 结构抑制热传导成为趋势. 一方面, 大孔隙率材料具有 较低的密度, 极易浮于水面, 能够与水体进行物理隔离, 将光热转换、热能的释放局限在气液分界面上极薄的 水层中. 另一方面, 这些材料自身都具有较低的热导率, 抑制了自上而下向水体的热量传播. 上海交通大学邓涛 课题组 ${ }^{[30]}$ 测得的无尘纸的热导率为 $0.48 \mathrm{~W} \mathrm{~m}^{-1} \mathrm{~K}^{-1}$, 低 于水体自身的热导率 $0.556 \mathrm{~W} \mathrm{~m}^{-1} \mathrm{~K}^{-1}$. 热场的数值模 拟表明, 无尘纸结构能够将气液界面附近的局部温度 提高约 $26^{\circ} \mathrm{C}$ (图5(c)).

影响热学调控的另一个重要因素——吸收体的辐 射损耗, 可以通过合理调控等离激元模式得到抑制. 通 过设计构建杂化模式, 可灵活调节等离激元的吸收带 边，实现选择性吸收，从而将吸收体的黑体辐射降低 到最低水平. 目前在热光伏领域, 等离激元吸收体已 被广泛应用，光栅、纳米颗粒链等金属微纳结构都被 证明具有优异的吸收截断能力 ${ }^{[39,40]}$. 在光蒸汽转换中, 南京大学朱嘉课题组 ${ }^{[31]}$ 证实多孔氧化铝模板的孔隙 与吸收光谱的带边直接相关. 其物理机制是由于金纳 米颗粒在 $\mathrm{AAO}$ 中的沉积形成等效纳米管结构, 而纳米 管的孔径变化带来了吸收截止频率的移动(图5(d)).

\section{4 等离激元光蒸汽的应用}

随着等离激元和光热转换领域的不断发展，结合 微纳加工技术, 等离激元光蒸汽转换已逐步应用于多 个能源领域. 图6展示了当今等离激元光蒸汽转换的 最新应用成果.

\section{1 海水淡化}

清洁的水资源对于人类生产生活至关重要. 据估 计，到 2050 年，全球将有约 50 亿人缺乏清洁的水资源 供给. 而带有高盐度的海水, 占据了全球水资源的 $96.53 \%$. 这部分水资源若得到充分利用，将大幅缓解 当今资源紧缺的形势. 传统的反渗透膜工艺, 存在造 

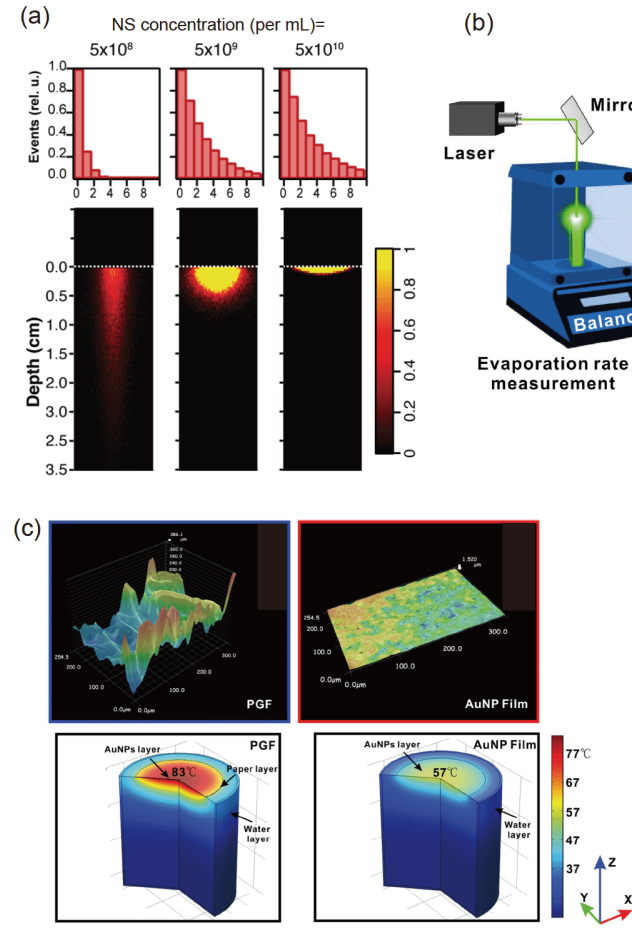

(b)
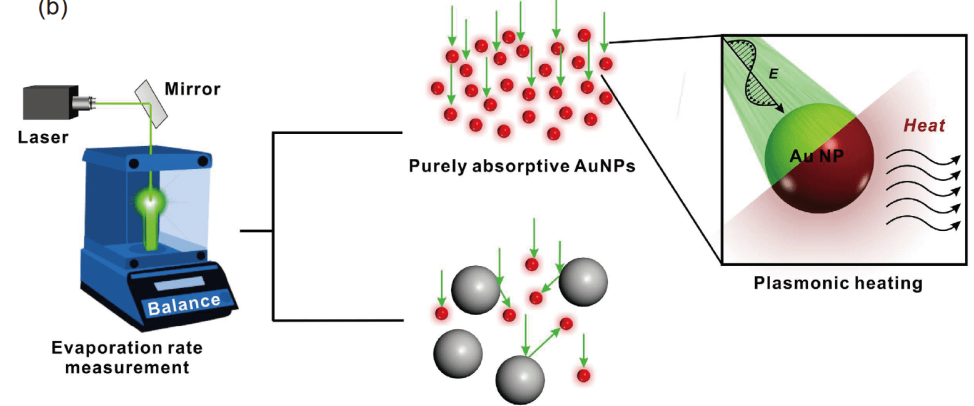

Plasmonic heating

(d)

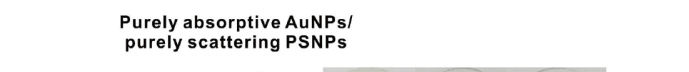

图 5 (网络版彩图)等离激元光蒸汽转换的热学调控过程. (a) 随着金纳米壳浓度的增加, 光子的内部散射次数普遍增加, 并且 吸收过程愈发向水-空气界面聚集 ${ }^{[37]}$. (b) 单纯吸收的金纳米颗粒, 和单纯散射的PSNP纳米颗粒. 该角色的分工, 能够比单一的 金纳米颗粒实现更强的吸收效果, 以及更高度的热学局域 ${ }^{[38]}$. (c) $2 \mathrm{D}$ 衬底无尘纸的使用(左)能够比单纯的纳米金薄片(右)实现 更高的表面温度 $\left(\Delta T \sim 26^{\circ} \mathrm{C}\right)$ 和较低的热传导耗散 ${ }^{[30]}$. (d) AAO 孔洞半径的变化, 能够创建不同尺寸的纳米同心柱结构, 实现吸 收带边的移动 ${ }^{[31]}$

Figure 5 (Color online) Thermal management for plasmonic-based solar evaporator. (a) With the increase in the concentration of gold nanoshells, internal scattering numbers for incident photons generally increase, and the absorption process becomes more localized towards the liquid-air interface [37]. (b) Purely absorbing gold nanoparticles, and purely scattering PSNP nanoparticles. Division of responsibility enables enhanced absorptivity and localization effects [38]. (c) Airlaid paper, a 2D substrate, gives rise to higher surface temperature $\left(\Delta T \sim 26^{\circ} \mathrm{C}\right)$ and lower heat conduction loss [30]. (d) Changes in size of the AAO pores result in size-varying concentric nanocylinders. A tunable bandwidth is therefore created, paving ways for convenient radiation control [31].

价高、体系庞大、耗电量大等问题, 不适用于偏远地 区的水质净化. 通过太阳能蒸发海水, 并对蒸汽进行 冷凝收集, 是一种相对更为可行的方法.

从成本上考虑, 南京大学朱嘉课题组 ${ }^{[33]}$ 提出基于 金属铝的等离激元吸收体的海水淡化方案. 他们使用 廉价的铝纳米颗粒进行三维自组装, 得到的AlNPAAM薄膜通过光蒸汽实验, 能够将海水的盐度降低4 个数量级. 蒸发纯化得到的水质, 超过WHO, EPA等机 构提出的饮用水水质标准. 尽管此AINP-AAM结构从 功效上看, 与反渗透膜工艺还存在一定差距, 但无需 额外的能量输入, 并且具有良好的便携性, 比较适用 于微型海水淡化装置.

此外, 还可以围绕等离激元光蒸汽体系构建多用 途光热器件, 在淡化海水的同时也可兼顾光解水和离
子发电等. $\mathrm{Ho}$ 课题组 ${ }^{[41]}$ 将 $\mathrm{Ag} @ \mathrm{TiO}_{2}$ 核-壳纳米颗粒沉 积于 $\mathrm{SiO}_{2}$ 基质, 能够同时实现海水淡化和光解水. 纳 米银颗粒通过LSP效应吸收入射光, 为两种反应提供 热量来源. 热能转变为蒸发的焓变, 在蒸汽冷凝后得 到淡水; 同时海水中的氯离子在空穴的激发下转化为 自由基, 使部分氢离子转化为双原子分子, 从而制备 出氢气.

\section{2 蒸汽灭菌}

对于光热转换产生的高温蒸汽, 海水淡化选择了 冷凝收集的方式, 蒸汽所携带的能量则往往被浪费. Neumann等人 ${ }^{[42]}$ 针对此能量设计了太阳能高压锅系 统, 将其应用于灭菌领域. 他们采用金纳米流体, 分别 设计出闭环的便捷式医疗灭菌装置和开环的大型生活 
污秽处理系统, 能够有效杀灭病原体.

在灭菌系统中, 有两个重要的指标 ${ }^{[33]}:$ (1) 蒸汽的 响应速度. 蒸汽的快速响应, 使得系统能够在短时间内 建立多个蒸汽灭菌周期, 提高灭菌效率. (2) 蒸汽温度 和压力. 蒸汽自身的高温和极高的饱和蒸气压, 都能够 有效消灭病原体．等离激元的快速热响应和局域热点 分布特性，非常贴合蒸汽杀菌的需求. Neumann等 人 $^{[42]}$ 设计的装置，由于有效抑制了对体块水的加热 $(<\sim 20 \%)$ ，实现了热能的集中利用. 以开环系统为例， 在 $75 \mathrm{~min}$ 内达到 $132^{\circ} \mathrm{C}$ 的高温, 并能够在 $5 \mathrm{~min}$ 内完成 一次灭菌.

在以往的研究中, 光蒸汽转换多以碳基材料为吸 收体. 例如, 近年来通过收集蒸汽液化能量发电 ${ }^{[44]}$, 或 是利用海水在热蒸发体系中的离子梯度差异发电 ${ }^{[45]}$, 都是选择了石墨烯、碳纳米管等碳基材料，而关于等 离激元吸收体的应用尚不多见．未来这些领域上的空 白, 值得科研工作者更多的关注.

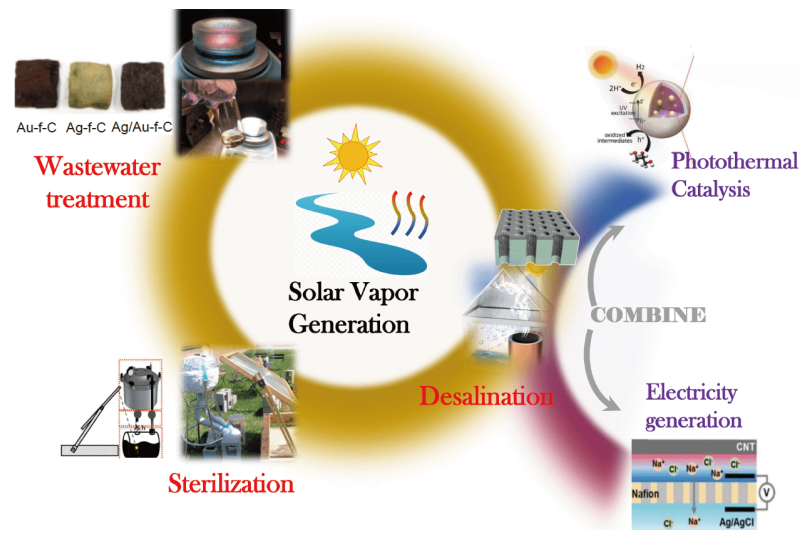

图 6 (网络版彩图)光蒸汽转换的不同应用: 海水淡化 ${ }^{[33]}$ 、 污水处理 ${ }^{[42]}$ 、高温灭菌 ${ }^{[42]}$ 等. 其中海水淡化与光热催化、 离子梯度势发电等海水利用正呈现出紧密结合的趋势, 多功 能的光热利用系统逐步被开发出来 ${ }^{[41,45]}$.

Figure 6 (Color online) Applications of photothermal conversion: desalination [33], wastewater treatment [42] and high-temperature sterilization [42]. Among them, desalination is showing an intensive trend for combination with other photothermal applications, such as photothermal catalysis and ion-gradient induced electricity generation. Multifunctional photothermal systems are gradually being invented $[41,45]$.

\section{5 总结与展望}

本文首先介绍了等离激元吸收体的原理和光蒸汽 转换的评判标准, 接着围绕光学吸收、热学调控两个 核心概念，总结了等离激元光蒸汽系统的构建思路. 经过科研工作者近十年的探索，等离激元光蒸汽系统 的光吸收效率已经能够达到 $99 \%$ ，在热损失的抑制上 也进行了较为系统的研究.

和碳基材料相比，等离激元材料的光蒸汽转换需 要着重考虑成本、水通道设计等方面. 现阶段仍存在 的以下主要问题.

(1) 光热转换的微观机理限制了我们对光蒸汽转 换系统的理解，仍有待探索和发展. 在过去的几十年 中，等离激元现象已经从微观角度被清晰、定量地阐 释. 等离激元和光热转换微观图像间的衔接, 能够让 我们进一步认识光蒸汽转化过程, 是一条可行且必要 的道路.

(2) 供水通道的设计有待完善. 水通道设计一直是 与光学吸收、热学调控相提并论的关键模块. 合理的 水通道设计，在供给充分的蒸发水源的同时，也几乎 隔绝了结构与水体的直接接触. 在基于碳基材料的光 蒸汽系统中, 我们看到很多出色的 $2 \mathrm{D}^{[46]}$ 乃至 $1 \mathrm{D}^{[1]}$ 结构 的水通道构建方案. 而围绕等离激元结构的文献中, 水 通道的构建往往是通过第3节所述的多孔祄底直接实 现; 这类结构向体块水的传导热损失依然有待解决.

（3）许多文献虽然实现了延伸至数个微米的宽带 吸收，但对于波长大于 $4 \mu \mathrm{m}$ 的波段的吸收机理，却没 有明确分析. 这是因为当电磁波波长大于 $4 \mu \mathrm{m}$ 时, 可 能的光吸收机理非常多, 除了等离激元吸收外, 还有 分子转动振动吸收、杂质吸收、自由载流子吸收、晶 格振动吸收等等, 难以严格区分出等离激元吸收的贡 献. 寻找更干净的等离激元微纳结构体系, 可能是未 来深入研究等离激元光热机理的突破口之一.

尽管存在诸多亟待解决的问题，等离激元材料仍 因光谱可调、亚波长电磁场局域、快速热响应等优异 的光学、热学特征, 在光蒸汽转换中具有良好的发展 前景, 值得进一步的研究.

\section{参考文献}

1 Li X, Lin R, Ni G, et al. Three-dimensional artificial transpiration for efficient solar waste-water treatment. Natl Sci Rev, 2018, 5: 70-77 
2 Bae K, Kang G, Cho S K, et al. Flexible thin-film black gold membranes with ultrabroadband plasmonic nanofocusing for efficient solar vapour generation. Nat Commun, 2015, 6: 10103

3 Kiriarachchi H D, Awad F S, Hassan A A, et al. Plasmonic chemically modified cotton nanocomposite fibers for efficient solar water desalination and wastewater treatment. Nanoscale, 2018, 10: 18531-18539

4 Wood R W. On a remarkable case of uneven distribution of light in a diffraction grating spectrum. Proc Phys Soc London, 1901, 18: 269-275

5 Fano U. Some theoretical considerations on anomalous diffraction gratings. Phys Rev, 1936, 50: 573

6 Ebbesen T W, Lezec H J, Ghaemi H F, et al. Extraordinary optical transmission through sub-wavelength hole arrays. Nature, 1998, 391: 667-669

7 Stockman M I, Bergman D J. Surface plasmon amplification through stimulated emission of radiation (SPASER). In: Proceedings Volume 5218, Complex Mediums IV: Beyond Linear Isotropic Dielectrics. San Diego, 2003

8 Haynes C L, Van Duyne R P. Plasmon-sampled surface-enhanced Raman excitation spectroscopy. J Phys Chem B, 2003, 107: 7426-7433

9 Alù A, Engheta N. Plasmonic materials in transparency and cloaking problems: Mechanism, robustness, and physical insights. Opt Express, 2007, 15: $3318-3332$

10 Neumann O, Urban A S, Day J, et al. Solar vapor generation enabled by nanoparticles. ACS Nano, 2013, 7: 42-49

11 Zhou L, Tan Y, Ji D, et al. Self-assembly of highly efficient, broadband plasmonic absorbers for solar steam generation. Sci Adv, 2016, 2: e1501227

12 Liu C, Huang J, Hsiung C E, et al. High-performance large-scale solar steam generation with nanolayers of reusable biomimetic nanoparticles. Adv Sustain Syst, 2017, 1: 1600013

13 Boriskina S V, Ghasemi H, Chen G. Plasmonic materials for energy: From physics to applications. Mater Today, 2013, 16: 375-386

14 Maier S A. Plasmonics: Fundamentals and Applications. New York: Springer, 2007

15 Abass A, Le K Q, Alù A, et al. Dual-interface gratings for broadband absorption enhancement in thin-film solar cells. Phys Rev B, 2012, 85: 115449

16 Zhang J, Bai W, Cai L, et al. Omnidirectional absorption enhancement in hybrid waveguide-plasmon system. Appl Phys Lett, 2011, 98: 261101

17 Farooq S, de Araujo R E. Engineering a localized surface plasmon resonance platform for molecular biosensing. Open J Appl Sci, 2018, 8: 126139

18 Fan X, Zheng W, Singh D J. Light scattering and surface plasmons on small spherical particles. Light Sci Appl, 2014, 3: e179, arXiv: 1407.2345

19 Sprünken D P, Omi H, Furukawa K, et al. Influence of the local environment on determining aspect-ratio distributions of gold nanorods in solution using Gans theory. J Phys Chem C, 2007, 111: 14299-14306

20 Taflove A, Hagness S C. Computational Electrodynamics: The Finite-Difference Time-Domain Method. 3rd ed. Boston, MA: Artech House, 2005

21 Linic S, Christopher P, Ingram D B. Plasmonic-metal nanostructures for efficient conversion of solar to chemical energy. Nat Mater, 2011, 10: 911-921

22 Zhang H, Zhang R, Schramke K S, et al. Doped silicon nanocrystal plasmonics. ACS Photon, 2017, 4: $963-970$ Prodan E, Radloff C, Halas N J, et al. A hybridization model for the plasmon response of complex nanostructures. Science, 2003, 302: 419-422 Zhao Y, Pan H, Lou Y, et al. Plasmonic $\mathrm{Cu}_{2-x} \mathrm{~S}$ nanocrystals: Optical and structural properties of copper-deficient copper(I) sulfides. J Am Chem Soc, 2009, 131: 4253-4261

25 Brongersma M L, Halas N J, Nordlander P. Plasmon-induced hot carrier science and technology. Nat Nanotech, 2015, 10: 25-34 Bashevoy M V, Fedotov V A, Zheludev N I. Optical whirlpool on an absorbing metallic nanoparticle. Opt Express, 2005, 13: 8372-8379 Bohren C F. How can a particle absorb more than the light incident on it? Am J Phys, 1983, 51: 323-327

30 Liu Y, Yu S, Feng R, et al. A bioinspired, reusable, paper-based system for high-performance large-scale evaporation. Adv Mater, 2015, 27: $2768-2774$

31 Zhou L, Zhuang S, He C, et al. Self-assembled spectrum selective plasmonic absorbers with tunable bandwidth for solar energy conversion. Nano Energy, 2017, 32: 195-200

32 Zhu M, Li Y, Chen F, et al. Plasmonic wood for high-efficiency solar steam generation. Adv Energy Mater, 2018, 8: 1701028 
33 Zhou L, Tan Y, Wang J, et al. 3D self-assembly of aluminium nanoparticles for plasmon-enhanced solar desalination. Nat Photon, 2016, 10: 393398

34 Zhang L, Xing J, Wen X, et al. Plasmonic heating from indium nanoparticles on a floating microporous membrane for enhanced solar seawater desalination. Nanoscale, 2017, 9: 12843-12849

35 Ishii S, Sugavaneshwar R P, Nagao T. Titanium nitride nanoparticles as plasmonic solar heat transducers. J Phys Chem C, 2016, 120: 2343-2348 Ma C, Yan J, Huang Y, et al. The optical duality of tellurium nanoparticles for broadband solar energy harvesting and efficient photothermal conversion. Sci Adv, 2018, 4: eaas9894

37 Hogan N J, Urban A S, Ayala-Orozco C, et al. Nanoparticles heat through light localization. Nano Lett, 2014, 14: 4640-4645

38 Zhao D, Duan H, Yu S, et al. Enhancing localized evaporation through separated light absorbing centers and scattering centers. Sci Rep, 2015, 5: 17276

39 Wu C, Neuner III B, John J, et al. Metamaterial-based integrated plasmonic absorber/emitter for solar thermo-photovoltaic systems. J Opt, 2012, 14: 024005

40 Wang X, Li H, Yu X, et al. High-performance solution-processed plasmonic Ni nanochain- $\mathrm{Al}_{2} \mathrm{O}_{3}$ selective solar thermal absorbers. Appl Phys Lett, 2012, 101: 203109

41 Gao M, Connor P K N, Ho G W. Plasmonic photothermic directed broadband sunlight harnessing for seawater catalysis and desalination. Energy Environ Sci, 2016, 9: 3151-3160

42 Neumann O, Feronti C, Neumann A D, et al. Compact solar autoclave based on steam generation using broadband light-harvesting nanoparticles. Proc Natl Acad Sci, 2013, 110: 11677-11681

$43 \mathrm{Li} \mathrm{J}, \mathrm{Du}$ M, Lv G, et al. Interfacial solar steam generation enables fast-responsive, energy-efficient, and low-cost off-grid sterilization. Adv Mater, 2018, 30: 1805159

$44 \mathrm{Li} \mathrm{X,} \mathrm{Li} \mathrm{J,} \mathrm{Lu} \mathrm{J,} \mathrm{et} \mathrm{al.} \mathrm{Enhancement} \mathrm{of} \mathrm{interfacial} \mathrm{solar} \mathrm{vapor} \mathrm{generation} \mathrm{by} \mathrm{environmental} \mathrm{energy.} \mathrm{Joule,} \mathrm{2018,} \mathrm{2:} \mathrm{1331-1338}$

Yang P, Liu K, Chen Q, et al. Solar-driven simultaneous steam production and electricity generation from salinity. Energy Environ Sci, 2017, 10: 1923-1927

46 Li X, Xu W, Tang M, et al. Graphene oxide-based efficient and scalable solar desalination under one sun with a confined 2D water path. Proc Natl Acad Sci, 2016, 113: 13953-13958 


\title{
Plasmonic nanostructures for advanced interfacial solar vapor generation
}

\author{
LIU HaiZhou ${ }^{1}$, YU XiaoQiang ${ }^{2}$, LI JinLei ${ }^{1}$, XU Ning ${ }^{1},{\text { ZHOU } \text { Lin }^{1 *}}^{{ }^{*}}$ \& ZHU Jia ${ }^{1 *}$ \\ ${ }^{1}$ College of Engineering and Applied Sciences, Nanjing University, Nanjing 210093, China; \\ ${ }^{2}$ School of Physics, Southeast University, Nanjing 211189, China
}

With the development of artificial microstructures and photothermal conversion, solar vapor generation (SVG) has become one of the most exciting fields of solar thermal energy utilizations, among which plasmonic photothermal evaporators have garnered tremendous interests. As the most important components of solar evaporators, plasmonic microstructures possess at least three appealing photothermal advantages with respect to traditional solar-thermal conversion devices. Firstly, plasmonic microstructures based solar absorbers can achieve highly efficient light harvesting across the entire solar irradiance spectral range with weighted solar absorptivity up to $95 \%$ or even higher. Secondly, plasmonic absorbers possess unique local heating effects which originate from both the material level (plasmonic hot spots effect) and system level (interfacial heating effect). Thirdly, plasmonic microstructures can exhibit tunable absorption and thermal emission properties in full wavelength range (from ultraviolet to mid-infrared), ideal for effective thermal loss suppression. In addition, plasmon-based solar vapor generation is also featured by a couple of pronounced materials and/or chemical priorities, such as effective anti-corrosiveness, strong recyclability as well as fast thermal response, which make them ideal candidates for some point-of-use off-grid applications, such as solar desalination for high salt concentration, wastewater treatment, domestic solar sterilization, etc. Aiming at the recent progresses on plasmon assisted efficient solar evaporation, this review demonstrates the photothermal designs on the emerging plasmonic solar vapor generation systems, which mainly focus on the microstructures enabled nanophotonic designs and thermal manipulation. Finally, the plasmonic solar evaporation based photothermal applications and a couple of crucial issues for the future prospective are outlined as well.

plasmonic resonance, solar vapor generation, nanostructures, solar energy

PACS: $64.70 . \mathrm{Fx}, 65.80 .+\mathrm{n}$, 78.67.Bf, 89.30.Cc

doi: 10.1360/SSPMA-2019-0070 